\title{
The role of sound propagation in concentrated colloidal suspensions
}

\author{
A. F. Bakker \\ Computational Physics, Faculty of Applied Sciences, Delft University of Technology, Lorentzweg 1, \\ 2628 CJ Delft, The Netherlands \\ C. P. Lowe ${ }^{\text {a) }}$ \\ Department of Chemical Engineering, University of Amsterdam, Nieuwe Achtergracht 166, \\ 1018 WV Amsterdam, The Netherlands
}

(Received 30 October 2001; accepted 7 January 2002)

\begin{abstract}
In a suspension, the hydrodynamic interactions between particles can propagate by two mechanisms: relatively slowly, by the diffusion of transverse momentum, or relatively rapidly, by the propagation of sound waves. Here we describe computer simulation results for the collective and single particle dynamics of colloidal particles with the aim of clarifying the role of sound. We find that for single particle motion the effect is rather trivial. As for an isolated particle, compressibility modifies the decay of velocity fluctuations only at very short times. For collective correlations this is not true. Our results show that the multiple scattering of sound waves between particles can induce correlated collective motions on time scales comparable with the diffusion of transverse momentum. The effects of compressibility are no longer restricted to very short times and manifest themselves as rapid oscillations in the time dependence of the collective diffusion coefficient. We suggest that these oscillations can largely be explained in terms of "effective" incompressible hydrodynamic theory, the suspension bulk viscosity, kinematic viscosity, and speed of sound becoming the relevant parameters. The oscillations are furthermore centered on the (hypothetical) incompressible result. Thus, while the effects of sound propagation may extend to surprisingly long times, the net effect remains limited to very short times. We discuss where these sound-induced oscillations will be relevant experimentally. (C) 2002 American Institute of Physics.
\end{abstract}

[DOI: 10.1063/1.1454995]

\section{INTRODUCTION}

A colloidal system consists of particles that are atomically large (the colloidal particles) dispersed in a solvent to form a liquid-like dispersion. In concentrated suspensions there are interactions between the colloidal particles that, relative to the dilute case, modify the dynamics. These "hydrodynamic" interactions are caused by colloidal particles influencing one another by momentum transfer through the fluid occupying the space between them. This transport of momentum can take place by two mechanisms that generally operate on very different time scales. The first is the diffusion of transverse momentum. The second is the propagation of a sound wave longitudinal to the direction of motion.

Theoretical approaches developed thus far ${ }^{1,2}$ only take the first, diffusive, mechanism into account. Nonetheless, there are cases where the second, sonic, mechanism has been shown to be relevant. ${ }^{3,4}$ The suggestion can also been made that sound propagation may explain apparent discrepancies between computer simulations results and experiments that probe the time dependence of the hydrodynamic interactions in concentrated suspensions. ${ }^{5,6}$ With the development of novel experimental techniques, such as position correlation microscopy, it is possible to actually measure the timedependent hydrodynamic interactions between isolated colloidal particles. ${ }^{7}$ It is now possible, therefore, to study the

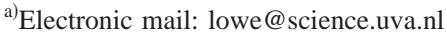

role of sound propagation in unprecedented experimental detail. In this paper our aim is to establish, from computer simulations, precisely what role we expect sound to play.

To begin with, it is useful to consider a case where considerable theoretical progress can be made, namely, a single spherical colloidal particle. This serves as a useful means of illustrating the relative characteristics of the two mechanisms. The analysis of the single particle case begins with the observation that the equation of motion for the colloidal particle takes the form of a generalized Langevin equation (GLE). ${ }^{8-10}$ To study the decay of velocity fluctuations, it is useful to consider the velocity autocorrelation function, $C(t)$. In terms of one component of the instantaneous velocity $v_{i}(t)$, the velocity autocorrelation function (VACF) is defined as

$$
C(t)=\left\langle v_{i}(0) v_{i}(t)\right\rangle .
$$

The solution to the GLE for the Laplace transform of the velocity autocorrelation function (denoted by the tilde), in terms of the transform variable $z$, reads

$$
\widetilde{C}(z)=\frac{\left\langle v_{i}^{2}\right\rangle}{z+\gamma(z) / m},
$$

where $m$ is the particle mass. Given the equations of motion describing the dynamics of the solvent and imposing the correct boundary conditions on the surface of the particle, $\gamma(z)$ can in principle be calculated. The simplest assumption is that the motion of the solvent can be described by the incom- 
pressible Navier-Stokes equations. For a sphere of radius $a$, in a fluid with a kinematic viscosity $\nu$ and density $\rho$, and assuming a stick boundary condition, the problem can be solved ${ }^{11}$ to yield, in terms of the dimensionless transform variable $z^{*}=z a^{2} / \nu$, an expression for the dimensionless VACF

$$
\frac{\nu \widetilde{C}\left(z^{*}\right)}{a^{2} C(0)}=\left[z^{*}+\frac{9 \rho^{*}}{2}\left(1+\frac{1}{9} z^{*}+\sqrt{z^{*}}\right)\right]^{-1},
$$

where $\rho^{*}$ is the ratio of the mass of fluid displaced by the particle to the mass of the particle itself, i.e., $\rho^{*}$ $=4 \pi a^{3} \rho / 3 m$. Equation (3) implies that, for a given value of $\rho^{*}$, the normalized VACF is a unique function of the dimensionless time $\tau_{\nu}=t \nu / a^{2}$. Values of $\tau_{\nu}$ of the order of unity characterize the time it takes transverse momentum to diffuse a distance the order of a particle radius. As this is controlled by the viscosity, we subsequently refer to it as the viscous time scale. A notable feature of Eq. (3) is that it predicts a discontinuous drop in the value of the normalized VACF from unity at $t=0$ to a value $\left(1+\rho^{*} / 2\right)^{-1}$ at an infinitesimal time later. For a neutrally buoyant particle $\left(\rho^{*}=1\right)$ the normalized VACF drops instantaneously to a value of $2 / 3$, reflecting the fact that $1 / 3$ of the momentum is carried away by sound propagation and that this happens instantaneously. This so-called "added mass" effect is an artifact of assuming that the fluid is incompressible. In practice fluids are not, of course, completely incompressible. Sound propagates on a time scale set by the speed of sound through the system. To allow for the finite speed of sound, one needs to proceed as above, but solve the more complex compressible NavierStokes equations. Despite the greater degree of complexity, this problem can still be solved. ${ }^{12}$ Again imposing a stick boundary condition, an expression can be derived for $\gamma(z)$ and hence the VACF. The full result is given in Ref. 12; here we will just summarize the salient points.

The solution involves two additional parameters associated with sound propagation, the speed of sound $c$ and the bulk viscosity $\nu_{B}$. These are most conveniently incorporated into the analysis by defining, in addition to the viscous time, a "sonic" time $\tau_{s}=t c / a$ and a viscosity ratio $\beta=\nu_{B} / \nu$. For most fluids the latter is of the order of unity. The sonic time is the time relative to the time it takes a sound wave to travel a distance of a particle radius. To get an idea of the two time scales involved, it is useful to consider real times in a "typical" suspension. We consider a typical system to be particles of radius $1 \mu$ dispersed in water at room temperature. This being the case, we have $\tau_{\nu} \sim 1$ when $t \sim 10^{-6} \mathrm{~s}$, whereas $\tau_{s}$ $\sim 1$ when $t \sim 10^{-9}$ s. The ratio of the two $\alpha=\tau_{\nu} / \tau_{s}=a c / \nu$ takes the value $\alpha \sim 1000$. In this case sound propagates momentum much more rapidly than does viscous diffusion. In terms of the viscous and sonic time scales, the principal characteristics of the VACF in a compressible fluid can now be summarized as follows (real times, where given, correspond to the typical system described above).

At short times, $t \ll c / a\left(t \ll 10^{-9} \mathrm{~s}\right)$, the normalized VACF depends only on $c / a$ and is a unique function of the dimensionless "sonic" time scale $\tau_{s}=t c / a$. This very rapid decay replaces the discontinuity found

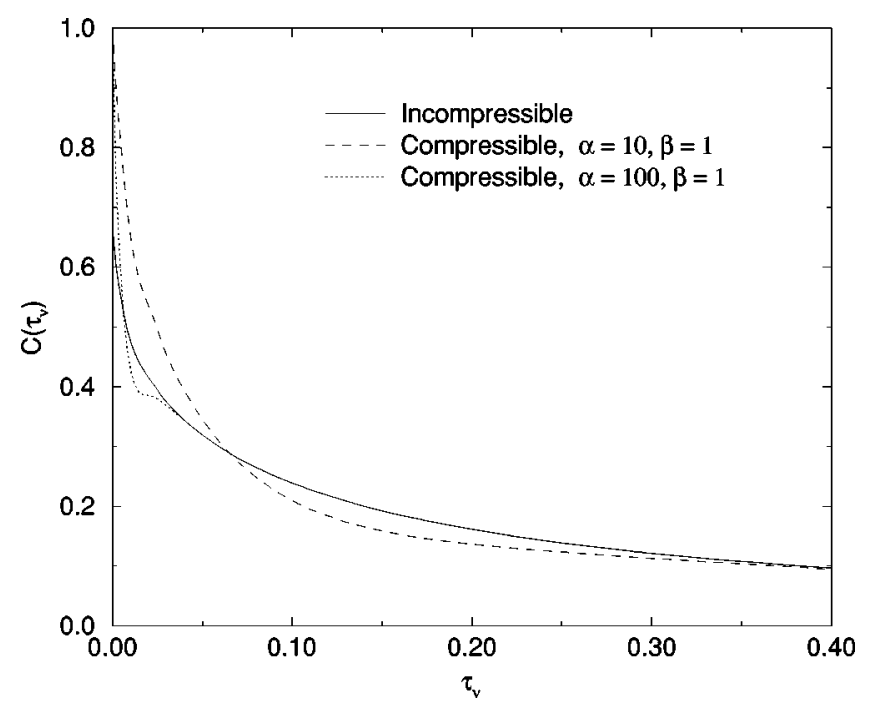

FIG. 1. The velocity autocorrelation function, $C\left(\tau_{\nu}\right)$ for a single colloidal sphere as a function of the dimensionless time $t \nu / a^{2}$. The solid line is the result for an incompressible fluid, the dashed and dotted lines are for a compressible fluid (parameters given in the legend).

for the incompressible case and reflects the period during which the particle generates the sound wave.

(ii) At intermediate times $t \sim a / c\left(t \sim 10^{-9}\right)$ there is a "mixed" region where the viscous time, sonic time, and viscosity ratio are all relevant. This reflects the period where the sound wave is separating from the particle.

(iii) For longer times $t>a / c\left(t>10^{-9}\right)$ the result reduces to the incompressible result above [Eq. (3)]. The sound wave has departed and consequently compressibility plays no role.

In summary, by going from an incompressible to a compressible model for the fluid, we get rid of the discontinuity in the VACF at very short times. Further, we find that for times $\tau_{s}>1$ we could have saved ourselves the trouble because the incompressible result suffices. This is illustrated in Fig. 1, where we have plotted the VACF predicted by compressible theory, for a neutrally buoyant particle with $\alpha=10$ and $\alpha$ $=100$, along with the incompressible result.

While the above discussion serves as a useful guide to the relevant parameters and time scales for a compressible fluid, it only applies for a single particle. What we are interested in is a concentrated suspension. The question then arises, what role does sound play in a suspension? In principle, part of the interactions can develop on the fast sonic time, rather than the slow viscous time. There are several reasons to establish whether such a mechanism is present. First, the separation of the relative time scales for processes operating in suspensions underlies theoretical treatments. A detailed discussion of this was given by Masters. ${ }^{13}$ Second, if sound can be neglected then the size of colloidal particles (at a given volume fraction) becomes essentially irrelevant. The results for any correlation function can be expressed as universal functions of the dimensionless parameters outlined above. On the other hand, if sound propagation does play a role this is no longer true. The size of the particles enters in 
the expression for the compressibility factor $\alpha$. Thus, if sound plays a role, correlation functions for particles of different sizes differ fundamentally. They cannot simply be scaled onto each other. This is important because the compressibility can vary considerably between different systems that could still lay claim to being called colloidal. For instance, we quoted a value $\alpha \sim 10^{3}$ for our typical system of a micron sized particle suspended in water. There is nothing special about 1 micron, though. Particles of sizes in the range $1 \mathrm{~nm}<a<10 \mu$ satisfy the criterion of being atomically large but macroscopically small. This implies compressibilities in the range $1<\alpha<10^{4}$, i.e., ranging from sound propagation and viscous diffusion occurring on similar time scales to the regime where the latter is much slower.

Despite the fact that sound propagation might be an important mechanism for propagating interactions in a suspension, until relatively recently the tacit assumption was that it is not. There are two substantive reasons to support this view. First, as we have seen, it is largely irrelevant for a single particle. Second, at sufficiently long times sound cannot play a role. This is simply because the integral of a correlation function is related to the zero frequency response. The zero frequency response to an external perturbation is the same for a compressible or an incompressible fluid. Thus, the speed of sound does not enter into the expression for the diffusion coefficient of a single particle, for example, whether the fluid is compressible or not. The question must always be one of when, not if, it becomes irrelevant. Experimental, theoretical, and computer simulation results have nonetheless suggested that, for concentrated suspensions, the effects of sound propagation may persist on longer time scales. First, experiments were reported ${ }^{14,15}$ probing the transient behavior of the mean-square displacement at short times $\left(\tau_{\nu} \sim 1\right)$. By comparing the short-time dynamics of particles in a concentrated suspension with those of an isolated particle, Zhu et al. ${ }^{14}$ found that the experimental data could be plausibly collapsed onto the single-particle curve if the time was rescaled in units of $a^{2} / \nu_{\phi}$, where $\nu_{\phi}$ is the kinematic viscosity of the suspension. This was surprising because, if the hydrodynamic interactions develop on the viscous time scale, one would expect that the suspension could only display this "effective fluid" behavior on time-scales $\tau_{\nu} \gg 1$. Computer simulations performed by $\operatorname{Ladd}^{16,17}$ appeared to confirm this, although more detailed simulations ${ }^{18}$ and theoretical work ${ }^{2}$ suggested that the effective fluid regime was only reached at longer times $\tau_{\nu} \gg 1$. Nonetheless, these observations led Español et al. ${ }^{19,20}$ to re-examine the role of sound propagation and suggest that the speed of sound may play a role in determining the time scale on which the hydrodynamic interactions propagate. Similarly, a discrepancy between experimental work and computer simulations vis á vis the collective dynamics, was tentatively ascribed to sound propagation. ${ }^{5}$ More recently, for a colloidal particle confined by some fixed geometry, it was demonstrated that the effects of sound propagation persist well beyond the sonic time scale and into the the viscous diffusion regime. ${ }^{3}$ In fact, compressibility actually determines the long-time form of the decay of the VACF.

Our aim here is thus to establish in what regimes the effect of sound propagation can be safely neglected and conversely where not. To do so, we have chosen to study the wave-vector-dependent current-current correlation function $J(k, t)$, defined as

$J(k, t)=\frac{1}{N}\left\langle\sum_{i=1}^{N} \sum_{j=1}^{N}\left[\hat{\mathbf{k}} \cdot \mathbf{v}_{i}(t) \mathbf{v}_{j}(0) \cdot \hat{\mathbf{k}}\right] \exp \left[i \mathbf{k} \cdot\left(\mathbf{r}_{i}-\mathbf{r}_{j}\right)\right]\right\rangle$,

where $k$ is the wave vector. The reasons for choosing to study this function are twofold. First, it gives an insight into collective correlations, that is, the ability of the instantaneous velocity of one particle to subsequently influence the motion of its neighbors. There is no analogue of this process for a single particle, so the inference that sound does not influence this function cannot, by analogy, be drawn. Second, the suggestion has already been made that this quantity is indeed influenced by sound propagation. ${ }^{5,6}$

\section{DESCRIPTION OF THE MODEL}

The model we have used consists of configurations of hard spheres, generated using standard Monte Carlo techniques, embedded in a model compressible fluid. In keeping with an assumption of short-time dynamics, we impose the time-scale separation $\tau_{\nu} \ll \tau_{p}$, where $\tau_{p}$ is a characteristic time for the particles to displace significantly. Thus, the positions of the colloidal particles are not considered to change during a run. The compressible fluid is modeled using a lattice Boltzmann equation, ${ }^{21}$ in which the state of the fluid system is characterized by the single-particle distribution function $n_{i}(\mathbf{r}, t)$ (see, e.g., Ref. 22). This denotes the average number of particles at a particular node of the lattice $\mathbf{r}$, at a time $t$, with the discrete velocity $\mathbf{c}_{i}$.

The motion of the colloidal particles is determined by the forces and torques exerted on them by the fluid. These are in turn a result of the stick boundary conditions applied at the solid/fluid interface. For moving boundaries the modified bounce-back rule is applied, whereby some of the particles moving in the same direction as the solid object are allowed to "leak" through, thus matching the fluid velocity to the object velocity at the boundary and implying (through conservation of total linear and angular momentum) a force and torque acting on the particle. Given this information, we can solve the equations of motion for the colloidal particles. To do so we use the "self-consistent" method, described in Ref. 23 , whereby the new fluid velocity at the boundary implies a force and torque on the object which, when incorporated into the equations of motion of the object, give the same new velocity for the particle. The advantage of this approach is that it is unconditionally stable. The density ratio can be chosen freely. This parameter, as we have seen, determines the relative proportion of momentum carried by sound propagation and momentum diffusion. If one is interested in the role of sound it is therefore important that it takes a physically sensible value $\rho^{*} \sim 1$. Because of stability problems, the simulations reported in Refs. 5 and 6 used a lower value of $\rho^{*}\left(\rho^{*} \leqslant 0.2\right)$, in which case the proportion of momentum carried away by sound waves is underestimated relative to the neutrally buoyant case. This was suggested as 
a possible source of discrepancy between the simulations and experimental results. For all the simulations we report here, $\rho^{*}$ takes the value unity.

The approach outlined above has been used to study (nominally) neutrally buoyant colloidal particles. ${ }^{18,23}$ However, there are certain artifacts generated by the presence of fluid inside what should be solid objects. ${ }^{24}$ We will be looking for the effects of sound propagation in a suspension largely by studying scaling behavior and these artifacts complicate the analysis considerably. We have therefore adopted the procedure outlined in Ref. 24 to remove the effects of the internal fluid. This basically involves setting all the distributions inside a particle to their equilibrium values for a fluid at rest. The force and torque required to do this are then incorporated as additional forces and torques in the equations of motion. This procedure can be shown to reproduce accurately the velocity autocorrelation function for a single spherical particle suspended in a compressible fluid. ${ }^{24}$

In order to calculate the wave-vector-dependent currentcurrent correlation function [Eq. (4)], we need to introduce velocity fluctuations into the system. One way to do so is to drive fluctuations in the stress tensor of the fluid and thus maintain the model colloidal particles at some prescribed temperature. ${ }^{21}$ The other is to impose velocity fluctuations on the colloidal particles and study the decay of these fluctuations in an otherwise dissipative system. ${ }^{25}$ According to Onsager's regression hypothesis the two methods should be equivalent. For reasons of computational expediency we have chosen the latter. Our method therefore proceeds as follows. The initial velocities, $\mathbf{v}_{i}(0)$, of the colloidal particles are generated from a Gaussian distribution with a fixed variance. The subsequent velocities $\mathbf{v}_{i}(t)$ are calculated by simply letting the system evolve in time as described above. The correlation function can then be calculated by simply substituting these quantities into Eq. (4) and taking the ensemble average over different initial configurations of particles and statistically independent initial values of the velocity. In practice we do both at the same time by repeating the calculation with different configurations, each with a set of initial velocities drawn independently from Gaussian distributions.

\section{RESULTS}

In order to separate out collective motions from single particle motion, it is useful to split the wave-vectordependent current-current correlation function into the (normal) velocity autocorrelation function, $C(t)$, and an "interaction" correlation function $J_{i}(k, t)$. Thus, we have $J(k, t)$ $=C(t)+J_{i}(k, t)$, where, by definition [see Eq. (4)] $J_{i}(k, t)$ is defined as

$J_{i}(k, t)=\frac{1}{N}\left\langle\sum_{i=1}^{N} \sum_{j \neq i}^{N}\left[\hat{\mathbf{k}} \cdot \mathbf{v}_{i}(t) \mathbf{v}_{j}(0) \cdot \hat{\mathbf{k}}\right] \exp \left[i \mathbf{k} \cdot\left(\mathbf{r}_{i}-\mathbf{r}_{j}\right)\right]\right\rangle$.

By considering the properties of $J(k, t)$ we can deduce some of the features we expect to characterize the $J_{i}(k, t)$. The integral over all times of $J(k, t)$ defines the wave vectordependent collective diffusion coefficient, $D(k)$. In the limit $k \rightarrow 0$ this should approach the (short-time) collective diffusion coefficient, $D_{c}$. In the opposite limit $k \rightarrow \infty, D(k, t)$ approaches the single-particle or self-diffusion coefficient. This is in turn equal to the integral over all time of the velocity autocorrelation function. In terms of transport coefficients, the time integral of $J_{i}(k, t)$ is therefore equal to the difference between the wave-vector-dependent collective diffusion coefficient and the self-diffusion coefficient, $D_{s}$

$$
\int_{0}^{\infty} J_{i}(k, t) d t=D(k)-D_{s} .
$$

Given the above, we expect that as $k \rightarrow \infty$ the integral of $J_{i}(k, t)$ will be zero, whereas in the limit $k \rightarrow 0$ it will be equal to $D_{c}-D_{s}$. The collective diffusion coefficient can be calculated (to a good approximation) theoretically ${ }^{26}$ and has also been calculated numerically. ${ }^{5,27}$ Both concur that $D_{c}$ $<D_{s}$, so we expect that, as $k \rightarrow 0$, the integral of $J_{i}(k, t)$ will be a negative quantity.

We will begin by considering the self-, or single-particle, contribution, $C(t)$. Measuring all quantities in units such that the lattice spacing and time step are equal to unity, we took a suspension with volume fraction $\phi=0.25$ and calculated the VACF for spheres with three different radii, $a$ $=2.5,4.5$, and 6.5 . The viscosity $\nu$, speed of sound $c$, and bulk viscosity $\nu_{B}$ were kept at constant values of $1 / 6,1 / \sqrt{2}$, and $1 / 30$, respectively. This means that for these three simulations the compressibility factor $\alpha(=a c / \nu)$ took the values 10.6, 19.1, and 36.0. For these three particle sizes we have been able to simulate systems large enough to calculate the VACF up to sufficiently long times, while still limiting the calculations to times less than those required for sound to cross the simulation box. The periodic boundary conditions applied at the faces of the simulation box cannot, therefore, influence the results (if such a procedure is not adopted there is a pronounced perturbation to the decay of the VACF as a particle gets hit by the sound wave generated by its periodic images).

The results we obtained for the normalized VACF, as a function of the viscous time scale $\tau_{\nu}$, are plotted in Fig. 2. Plotting the data in this form makes the effects of compressibility simple to spot. Any difference between the curves (apart from numerical errors, which are relatively small even for $a=2.5^{24}$ ) must be attributed to sound propagation. If the fluid is incompressible, then, in these dimensionless terms we are basically simulating the same system. Clearly, at short times the response of the three systems is not identical. However, at longer times the three functions converge, indicating that sound is playing no role. Furthermore, they become indistinguishable at shorter times as we decrease the compressibility (increase $\alpha$ ). More quantitatively, we find that the VACF calculated for $\alpha=10.6$ becomes indistinguishable from the other two (higher) values of $\alpha$ for $\tau_{\nu}>0.40$, whereas the result for $\alpha=19.1$ becomes indistinguishable from that calculated at $\alpha=36.0$ for times $\tau_{\nu}>0.20$. For the single-particle case, we saw that the effects of compressibility can be neglected on time scales long as compared to the sonic time $\tau_{s}$, that is $t>a / c$. In terms of the viscous time this condition corresponds to $\tau_{\nu}>1 / \alpha$, i.e., the effects of compressibility become negligible after a viscous time which 


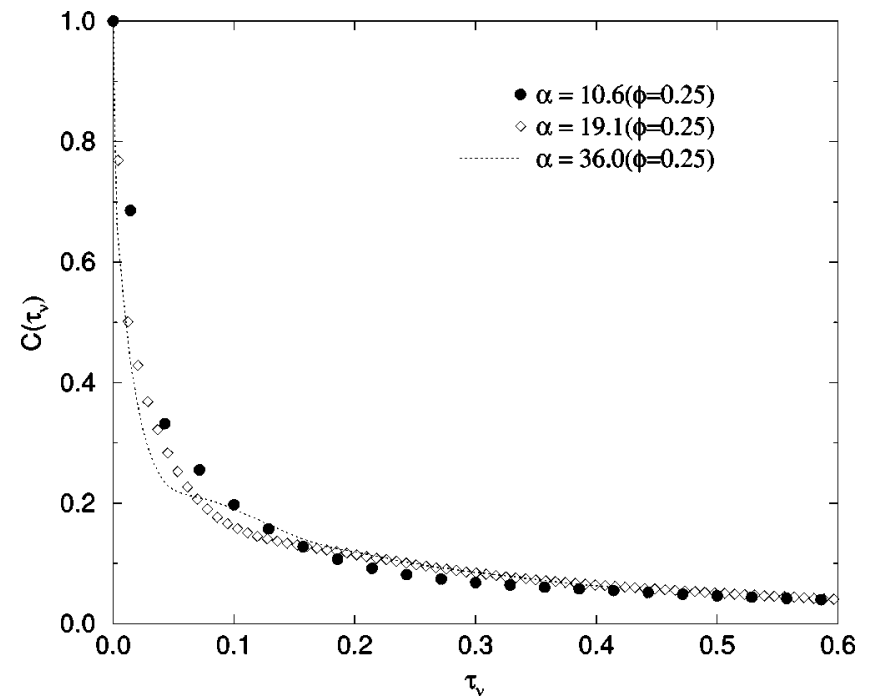

FIG. 2. The velocity autocorrelation function, $C\left(\tau_{\nu}\right)$, for a suspension of volume fraction $\phi=0.25$, as a function of the dimensionless time $t \nu / a^{2}$. The three sets of data correspond to three different values of the compressibility factor $\alpha$.

scales as $1 / \alpha$. The behavior we noted above is quite consistent with this being the case in a suspension. On increasing $\alpha$ from a value of 10.6 to 19.1 (a factor of 1.8), we see that the time at which incompressible behavior is recovered (the "incompressible" time) decreases by a factor of approximately 2. Given the uncertainty involved in establishing a precise value for the incompressible time, this difference cannot be regarded as significant. It is interesting to note that the incompressible time not only scales in the same way as for the single particle case but is also quantitatively almost identical. Examining the Bedeaux and Mazur result (Fig. 1) for a single particle, the incompressible time is, to a good approximation, $\tau_{\nu} \approx 4 / \alpha$. Extrapolating the same behavior to a suspension, one would predict an incompressible time of $\tau_{\nu}$ $=0.38$, for the system with $\alpha=10.6$, and $\tau_{\nu}=0.21$ for the system with $\alpha=19.1$. This compares with values 0.4 and 0.2 , respectively, calculated from the simulation results. The most obvious conclusion we can therefore draw from Fig. 2 is that, in a suspension, the influence of sound propagation on the velocity autocorrelation function is rather trivial. It is remarkably similar to the single-particle case in that it modifies the decay on the short sonic time scale. On the longer viscous time scale it is irrelevant and an incompressible theory could adequately describe the dynamics of the system.

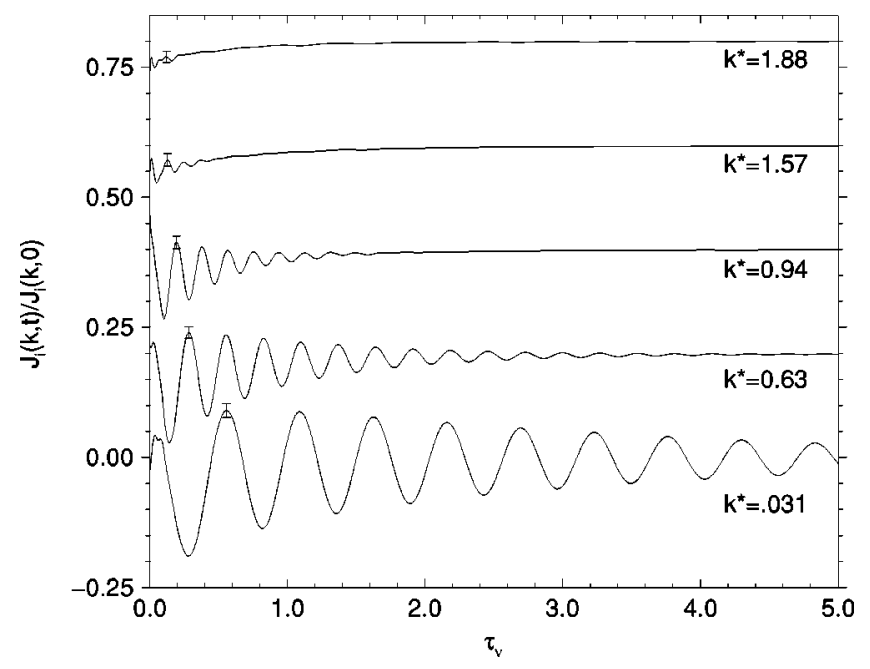

FIG. 3. The dimensionless interaction correlation function, $J_{i}(k, t) / J_{i}(k, 0)$, calculated for system $R 4$. The results for various values of the reduced wave vector $k^{*}$ are shown. In the interests of clarity, the data have been displaced, from bottom to top, by successive increments of 0.2 along the $y$ axis.

Turning our attention to the interaction part of the transverse current correlation function, $J_{i}(k, t)$, we found it useful to modify our approach in two respects. First, by comparing the results for simulations on different system sizes we found that, in contrast to the VACF, the periodic boundary conditions did not influence the results significantly, even on time scales long as compared to the time taken by a sound wave to cross the simulation box. We were therefore able to calculate $J_{i}(k, t)$, free from finite-size artifacts, up to longer times (and using smaller systems). Second, in order to gain a reasonable degree of insight into the role of sound propagation, the compressibility factor needed to be varied to a greater extent than was practical by varying the particle size alone. Six sets of simulations were carried out in total, all at a volume fraction $\phi=0.25$. The parameters associated with these simulations, which we denote $R 1, R 2, \ldots R 6$, are summarized in Table I. In Fig. 3 we have plotted $J_{i}(k, t)$, calculated for various values of the dimensionless wave vector $k^{*}=k a$, for run $R 4$. This simulation had a compressibility factor $\alpha=36$ and, as such, corresponds to particles of radius $a \sim 25 \mathrm{~nm}$ suspended in water, i.e., small colloidal particles. The most notable feature is clearly the pronounced oscillation. This phenomenon was also observed in computer simulations performed by Ladd et al. ${ }^{5}$ who further concluded that these oscillations were sonic in origin. However, these oscil-

TABLE I. The parameters associated with the six systems studied: sphere radius $(a)$, effective radius $\left(a^{*}\right)$, viscosity $(\nu)$, compressibility factor $(\alpha)$, sound wave attenuation coefficient $(\Gamma)$, number of spheres $(N)$, and system dimensions $(L)$. The volume fraction was $25 \%$ in all cases and values are quoted in lattice units.

\begin{tabular}{lccccccc}
\hline \hline Set & $a$ & $a^{*}$ & $\nu$ & $\alpha$ & $\Gamma$ & $N$ & $L \times L \times L$ \\
\hline$R 1$ & 1.5 & 1.5 & $1 / 6$ & 6.3 & 0.255 & 3820 & $60 \times 60 \times 60$ \\
$R 2$ & 2.5 & 2.5 & $1 / 6$ & 10.5 & 0.255 & 3820 & $100 \times 100 \times 100$ \\
$R 3$ & 4.5 & 4.5 & $1 / 6$ & 18.9 & 0.255 & 3820 & $180 \times 180 \times 180$ \\
$R 4$ & 8.5 & 8.5 & $1 / 6$ & 35.7 & 0.255 & 477 & $170 \times 170 \times 170$ \\
$R 5$ & 4.5 & 4.7 & $1 / 48$ & 157.9 & 0.0611 & 119 & $47 \times 47 \times 94$ \\
$R 6$ & 4.5 & 4.79 & $1 / 96$ & 321.89 & 0.0472 & 120 & $48 \times 48 \times 96$ \\
\hline \hline
\end{tabular}




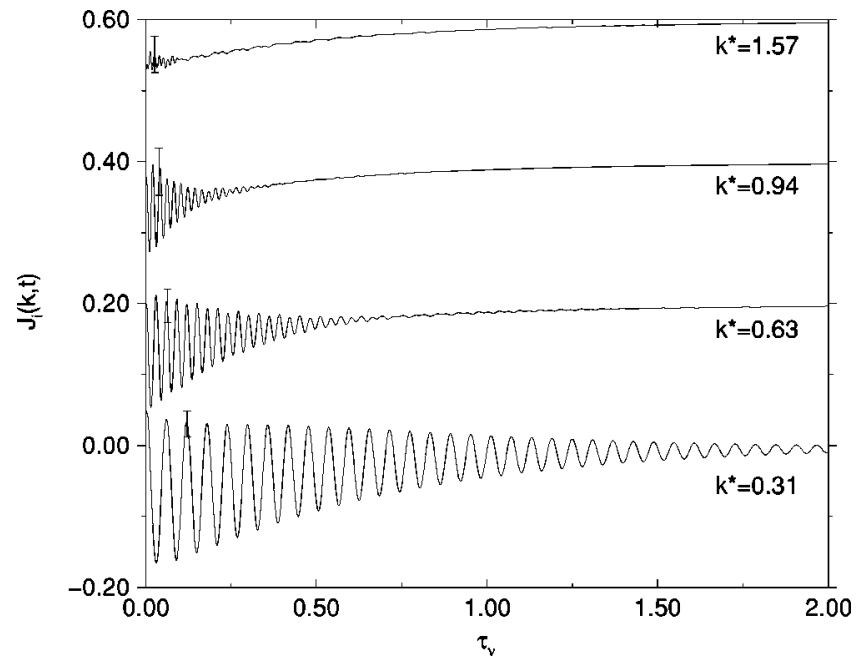

FIG. 4. As Fig. 3, but for run R6.

lations cannot be considered simply as artifacts of the model fluid being too compressible, ${ }^{5}$ as the results shown in Fig. 3 are parametrically correct for small colloidal particles. In Fig. 4 we show the equivalent plot for run $R 6$. For this simulation the compressibility factor was $\alpha=325$, approximately one order of magnitude greater. As such, it corresponds to particles of radius $a \sim 250 \mathrm{~nm}$ suspended in water. These could reasonably be described as "average" colloidal particles. As Fig. 4 shows, we still observe oscillations although they are characterized (at a given value of the dimensionless wave vector) by a higher frequency and more rapid damping than was the case for $\alpha=36$. Taking Figs. 3 and 4 together, it appears that these oscillations are sensitive to the compressibility of the system. The fact that their characteristics depend on the compressibility factor shows that they must be sonic in origin. If the solvent were incompressible this behavior could not be observed, and Figs. 3 and 4 would be identical. A further point illustrated by Figs. 3 and 4 is that both the frequency and damping of the oscillations depend on the wave vector. In order to come to any conclusion as to whether this effect will be relevant in the analysis of experimental results, it is necessary to establish the scaling with respect to the wave vector. There are two reasons for this. First, experimentally it is not possible to measure the correlation function $J(t)$ itself. It is only possible to measure its time integral (as in photocorrelation spectroscopy), or a quantity closely related to the time integral. The contribution these oscillations make to the integral of the correlation function, and the time scale over which this contribution will be significant, will depend on the amplitude, frequency, and rate of damping. Second, the time regime probed by experiments depends on the wave vector. Smaller wave vectors are, for instance, characterized by longer decay times for correlation functions of position. Thus, decreasing the wave vector normally implies probing longer times. If the oscillations in $J(k, t)$ increase in magnitude with decreasing wave vector, but correspondingly decay over shorter times, their effect may still not be relevant.

A convenient reference point for analyzing the wavevector-dependent current-current correlation function is what one may term "classic hydrodynamic" behavior. For a one-component, isothermal, compressible fluid, the contribution to $J_{i}(k, t)$ due to sound propagation, $J_{i}^{s}(k, t)$, is of the form $^{28}$

$$
J_{i}^{s}(k, t)=\frac{k_{B} T}{2 m \pi} \cos (k c t) \exp \left(-\Gamma k^{2} t\right)
$$

where $\Gamma=\left(4 \nu / 3+\nu_{B}\right)$ is the sound attenuation coefficient, $T$ the temperature, and $k_{B}$ Boltzmann's constant. The full form of $J_{i}^{s}(k, t)$, in the frequency domain, is given in Ref. 28 . Equation (7) is obtained by taking the inverse transformation for frequencies $\omega \ll c k$ and so can only be expected to hold for times $\tau_{s} k^{*} \gg 1$. In a suspension, $J_{i}^{s}(k, t)$ can in general be only one component of the total interaction function $J_{i}(k, t)$. We know this because, as noted above, the integral over all time of $J_{i}(k, t)$ at small $k$ is equal to the difference between the collective and self-diffusion coefficients. Furthermore, as we pointed out in the Introduction, this quantity, being the difference between transport coefficients, cannot depend on the compressibility of the fluid. There must therefore be an incompressible contribution to $J_{i}(k, t)$, i.e., a component that is independent of the compressibility of the solvent. This is clearly illustrated in both Figs. 3 and 4, particularly at short times, where $J_{i}(k, t)$ oscillates about a negative nonzero value. To try to separate out the compressible component of $J_{i}(k, t)$, it is convenient to work with the relative amplitudes of successive oscillations rather than absolute values. We therefore define the following quantities.

(i) A frequency $\omega$ defined as $\omega=2 \pi / \Delta t$, where $\Delta t$ is the time between successive maxima (or minima) in the oscillations. From the first maximum (or minimum) onwards we find that this value is constant, in agreement with Eq. (7). Equation (7) further predicts that $\omega=c k$.

(ii) A dimensionless "initial" amplitude $A_{0}^{*}$ $=A_{0} m / 2 \pi k_{B} T$, where $A_{0}$ is defined as the difference between the first minimum of $J_{i}(t)$ and the first maximum. According to Eq. (7), $A_{0}^{*}=\exp \left(-\Gamma k^{2} \pi / \omega\right)(1$ $+\exp \left(-\Gamma k^{2} \pi / \omega\right)$ ). If $k^{2} \Gamma \pi / \omega \ll 1$ (the decay of the oscillations is slow compared to the frequency), this can be expanded in the simpler form

$$
A_{0}^{*} \simeq 2-k^{2} \Gamma \pi / \omega+\mathcal{O} k^{4} .
$$

(i) A dimensionless time-dependent amplitude, $A^{*}\left(t_{l}\right)$, defined as

$$
A^{*}\left(t_{l}\right)=\left|J_{i}\left(t_{l}-\Delta t / 4\right)-J_{i}\left(t_{l}+\Delta t / 4\right)\right| m / 2 \pi k_{B} T,
$$

where $l=1,2, \ldots$, is an integer and $t_{l}=l \Delta t / 2+\Delta t / 4$. According to Eq. (7), this should decay as

$$
\begin{aligned}
2 A^{*}\left(t_{l}\right)= & \exp \left(-k^{2} \Gamma t_{l}\right)\left(\exp \left(-k^{2} \Gamma \pi / 2 \omega\right)\right. \\
& \left.+\exp \left(k^{2} \Gamma \pi / 2 \omega\right)\right) .
\end{aligned}
$$

Again, so long as $k^{2} \Gamma \pi / \omega \ll 1$, this can be expanded in a simpler form

$$
A^{*}\left(t_{l}\right) \simeq \exp \left(-k^{2} \Gamma t_{l}\right)+\mathcal{O} k^{4} .
$$




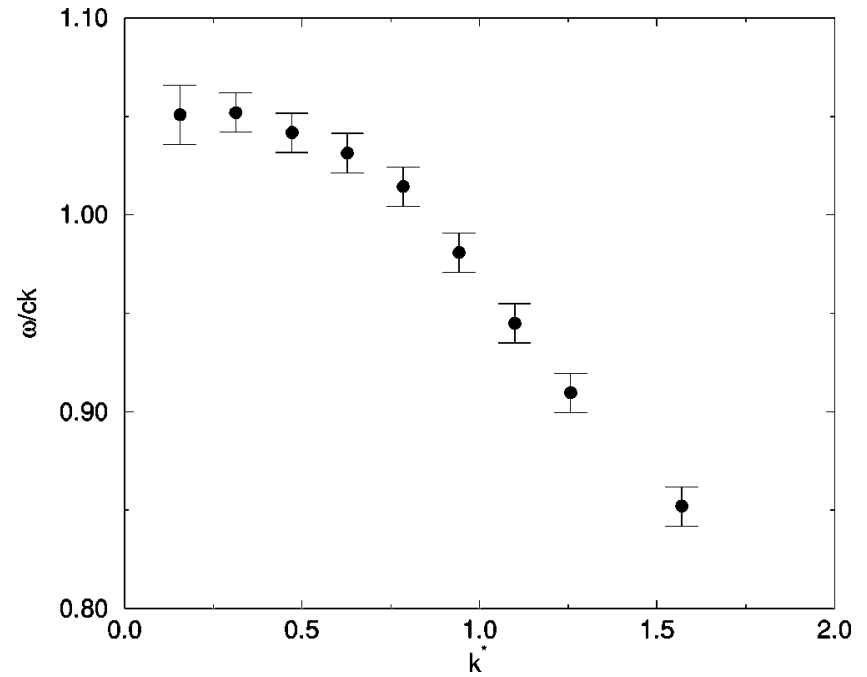

FIG. 5. The reduced frequency of the oscillations in the interaction correlation function as a function of the reduced wave vector $k^{*}=k a$. These data were obtained from system $R 4$. The other systems displayed the same behavior.

We will take these characteristics in turn and examine to what extent they describe the results we find in a suspension. Beginning with the frequency, in Fig. 5 we have plotted the dimensionless frequency, $\omega / c k$, as a function of the dimensionless wave vector. According to classic hydrodynamic theory, this function takes a value of unity, independent of wave vector. As Fig. 5 shows, the dimensionless frequency of the oscillations displays a weak dependence on the wave vector, but becomes independent of $k$ for $k^{*}<0.5$. That is, we recover hydrodynamic behavior on sufficiently long length scales. However, careful analysis of Fig. 5 shows that in this limit the dimensionless frequency is not asymptotically unity. The value we find is $\lim _{k \rightarrow 0} \omega / c k=1.05 \pm 0.01$. We now note, however, that there are two primary objections to applying the classic theory for a suspension. First, there is an ambiguity as to the values we should take for the transport coefficients. In the above analysis we have taken the speed of sound to be the speed of sound through the solvent. Of course, we do not necessarily expect the speed of sound in a suspension to be equal to the speed of sound in the solvent. Second, it takes no account of the structure of the fluid. One possible explanation for the low-frequency asymptote differing from unity concerns the former. It is simply that the speed of sound in the suspensions $c(\phi)$ is slightly greater than that in the solvent, specifically $c(\phi) / c=1.05$. We have independently confirmed that this is the case by measuring directly the speed of sound in the system (by examining the time at which sound-induced perturbations to the decay of the VACF, resulting from the periodic boundary conditions, are observed). This analysis also gives a value of $c(\phi) / c$ $=1.05$. The asymptotic behavior we observe for the frequency of the oscillations at long wavelengths is thus consistent with classic hydrodynamic theory if we replace a transport coefficient characteristic of the solvent, $c$, with a transport coefficient characteristic of the suspension, $c(\phi)$. If we now turn to the wave-vector dependence, this is most

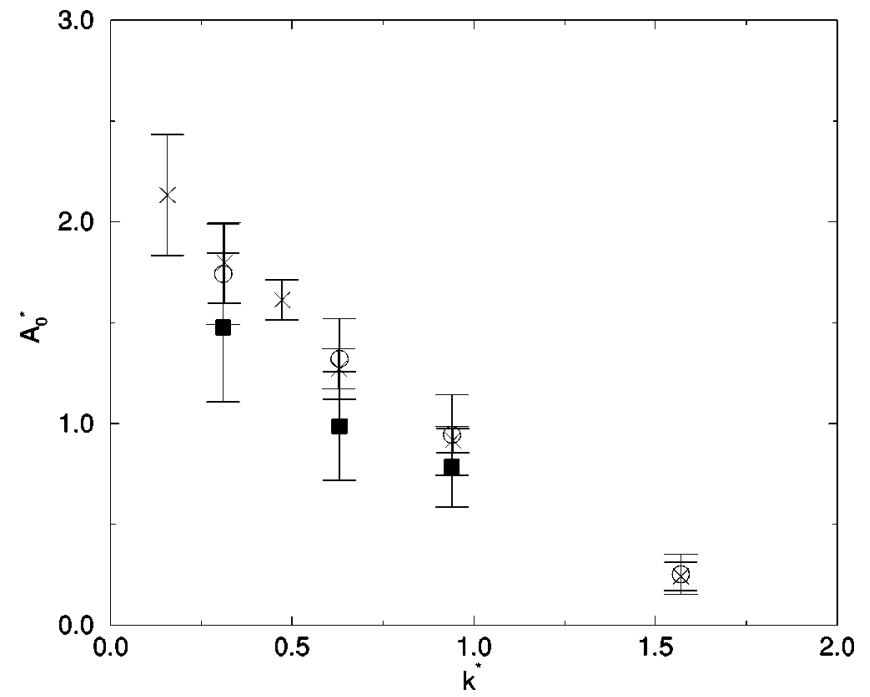

FIG. 6. The dimensionless initial amplitude of the oscillations in the interaction correlation function $A_{0} *$ (defined in the text) as a function of the reduced wave vector $k^{*}$. The data correspond to systems $R 2, R 4$, and $R 6$.

likely to be a consequence of the structure present in the fluid. This (in Fourier space) is most conveniently probed by the static structure factor $S(k)$

$$
S(k)=\frac{1}{N}\left\langle\sum_{i} \exp \left(i \mathbf{r}_{i} \cdot \mathbf{k}\right) \sum_{i} \exp \left(-i \mathbf{r}_{i} \cdot \mathbf{k}\right)\right\rangle .
$$

At sufficiently small wave vectors the static structure factor (SSF) approaches a constant value, reflecting the fact that, on sufficiently long length scales, the suspension appears homogeneous. For hard spheres at this volume fraction (see, for example, Ref. 29), the SSF becomes essentially independent of $k$ for $k^{*}<0.5$, in line with our estimate for the wave vectors for which we observe classic hydrodynamic behavior. Thus, the wave-vector dependence we observe in $\omega / c k$, at short wavelengths, is consistent with the structure of the suspension, not accounted for in the simple theory, playing a role. We finally note that generalizing the hydrodynamic result by defining a wave-vector-dependent speed of sound $c(k)$, such that $\omega / c(k) k=1$, the data we have plotted in Fig. 5 are consistent, at short wavelengths, with $c(k)<c$. That is, a wave-vector-dependent speed of sound less than the speed of sound in the solvent. Although this is somewhat counterintuitive, the same phenomenon-on length scales where structure cannot be neglected-is observed in simple atomic fluids ${ }^{30,31}$ and can be accounted for theoretically. ${ }^{32}$ It should also be pointed out that in simple atomic fluids the wavevector dependence of the speed of sound is significantly more pronounced. The suspensions we consider here follow classic hydrodynamic behavior to a better approximation than do simple fluids.

The second characteristic of classic hydrodynamic behavior, the initial dimensionless initial amplitude, $A_{0}^{*}$, is plotted in Fig. 6. Examining Fig. 6 we see that the dimensionless initial amplitude is approaching the value 2 as $k$ $\rightarrow 0$, so again we are recovering classic hydrodynamic behavior in the limit of long wavelengths. However, there is a clear $k$ dependence with the amplitude decreasing roughly linearly with increasing wave vector. The $k$ dependence is 


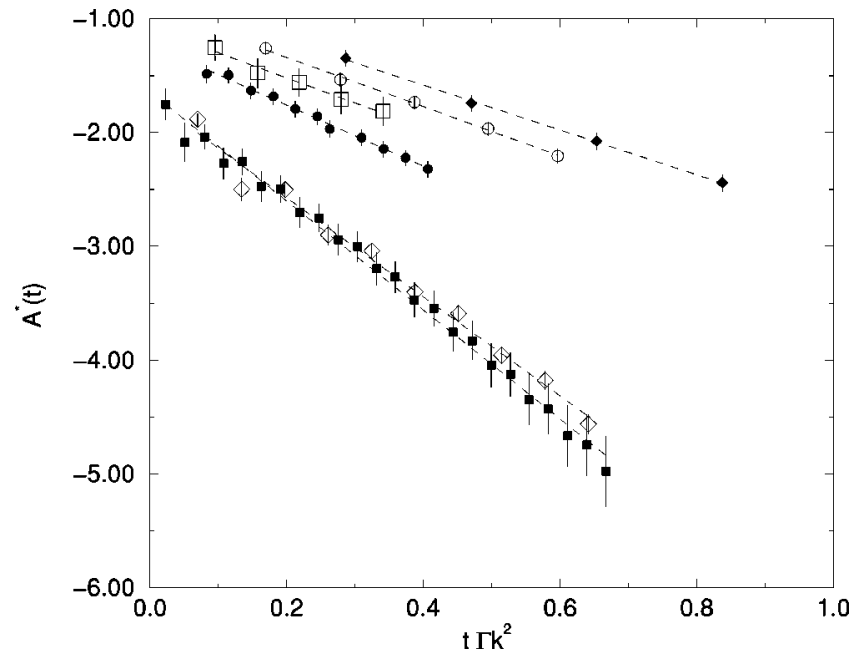

FIG. 7. Linear-log plot of the dimensionless time-dependent amplitude $A^{*}(t)$ of the oscillations in the interaction correlation function (defined in the text) as a function of the dimensionless time $t \Gamma_{0} k^{2}$. The reduced wave vector $k^{*}$ was equal to 0.314 . Data for all six systems are shown.

not quadratic, so it cannot be accounted for by the $\mathcal{O} k^{2}$ term appearing in Eq. (8) (which simply reflects the relatively trivial change in the value of the exponential decay envelope between the first minimum and first maximum). The $k$ dependence we observe therefore represents behavior not accounted for by simple hydrodynamics, again manifesting itself on length scales where the structure of the suspension cannot be neglected. One further point illustrated by Fig. 6 is that, following the trend, the amplitude of the oscillations tends to zero at a value $k^{*} \sim 2$. That is, we observe no collective effects at all for $k^{*}>1.75$. Presumably this reflects the fact that the minimum separation of $2 a$ between particles essentially defines a maximum wave vector at which particles interact via sound propagation. Again, the theory outlined above takes no account of this effect.

Having examined the factors determining the frequency and initial amplitude of the sound-induced oscillations, all that remains is to establish what determines the subsequent rate of decay. In Fig. 7 we have plotted, at a reduced wave vector $k^{*}=0.314$, the dimensionless time-dependent amplitude as a function of a dimensionless time $t \Gamma k^{2}$ for all six simulations. Hydrodynamically, this plot should be linear with a slope equal to unity. As Fig. 7 shows, the data are, to a good, approximation, linear. The amplitude of the oscillations decays exponentially. However, if we calculate the slopes by linear regression, the values are not unity. Following the same reasoning applied for the speed of sound, it seems sensible to interpret this as indicating that the sound wave attenuation coefficient in the suspension $\Gamma(\phi)$ is not the same as the solvent value $\Gamma$. That is, we expect the amplitude of the oscillations to decay as $\exp \left(-\Gamma(\phi) k^{2}\right)$. Having made this assumption, we can calculate values for $\Gamma(\phi)$ consistent with the data shown in Fig. 7. These values are summarized in Table II. As the table shows, for the first four systems $(R 1$, $R 2, R 3, R 4)$ the ratio $\Gamma(\phi) / \Gamma$ takes a roughly constant value between 2.0 and 2.6. For these simulations, the solvent viscosity is greater than the bulk viscosity so, to a first approximation, we can neglect the bulk viscosity and thus $\Gamma$
TABLE II. The apparent suspension sound wave attenuation coefficient, $\Gamma(\phi)$, normalized by the solvent value, $\Gamma$. The values are calculated from linear fits to the data shown in Fig. 7.

\begin{tabular}{cc}
\hline \hline Simulation & $\Gamma(\phi) / \Gamma$ \\
\hline$R 1$ & 2.1 \\
$R 2$ & 2.2 \\
$R 3$ & 2.0 \\
$R 4$ & 2.6 \\
$R 5$ & 4.8 \\
$R 6$ & 4.4 \\
\hline \hline
\end{tabular}

$\sim 4 \nu / 3$. If we further interpret $\Gamma(\phi)$ as depending on the suspension viscosity $\nu(\phi)$ and the suspension bulk viscosity $\nu_{B}(\phi)$, i.e., $\Gamma(\phi)=4 \nu(\phi) / 3+\nu_{B}(\phi)$, this condition corresponds to $\Gamma(\phi) / \Gamma=\nu(\phi) / \nu$ (so long as $\nu_{B}(\phi) / \nu_{B}$ $\leqslant \nu(\phi) / \nu)$. The value of $\nu(\phi)$ for suspensions of hard spheres is known.

Accurate multipole calculations performed by Ladd $^{33}$ give a value $\nu(\phi) / \nu=2.17$ at this volume fraction. The values we obtained for the ratio $\Gamma(\phi) / \Gamma[\sim \nu(\phi) / \nu]$ from the first four simulations are roughly equivalent to this value. It should be noted that because these simulations use spheres of different radii, the numerical errors are not the same. We expect the run $R 4$, where the radius of the spheres was 8.5 lattice units, to be the most accurate. This simulation gives a value $\Gamma(\phi) / \Gamma=2.6$, somewhat higher than the value for $\nu(\phi) / \nu$ quoted above. It is nonetheless clear from Table II that the most anomalous results are those for runs $R 5$ and $R 6$. These are characterized by, respectively, a solvent bulk viscosity greater than the kinematic viscosity and a bulk viscosity roughly equivalent to the kinematic viscosity. The obvious explanation for this is that the ratio $\nu_{B}(\phi) / \nu_{B}$ behaves significantly differently from the ratio $\nu(\phi) / \nu$. If we take the values of $\Gamma(\phi) / \Gamma$ for these two runs and solve for the two viscosity ratios, we find $\nu(\phi) / \nu=2.3$ and $\nu_{B}(\phi) / \nu_{B}=4$.8. If we now return to run $R 6$ but this time, instead of neglecting, we include the bulk viscosity, then, substituting this value for $\nu_{B}(\phi)$ we find that $\Gamma(\phi) / \Gamma=2.5$, in better agreement with the value we calculated. Taken as a whole, our results are therefore consistent with the sound wave attenuation coefficient in the suspension taking the form $\Gamma(\phi)=4 \nu(\phi) / 3$ $+\nu_{B}(\phi)$, if $\nu_{B}(\phi) / \nu_{B} \sim 4.8$, suggesting that the bulk viscosity of the suspension has a stronger dependence on the volume fraction than does the shear viscosity. It would be useful to test this hypothesis by calculating $\nu_{B}(\phi)$ directly. Unfortunately, we have not as yet succeeded in doing so. The above must therefore remain a working hypothesis.

We now turn our attention to the contribution the oscillations do, or do not, make to the time integral of $J_{i}(k, t)$. In Fig. 8 we have plotted the time integral of $J_{i}(k, t)$, at a reduced wave vector $k^{*}=0.314$, as a function of the viscous time scale. Data for five of the six runs are plotted; data from run $R 5$ is omitted for clarity (it follows the same trend). As the figure shows, for the data obtained from the least compressible simulation $(R 6)$, the frequency of the oscillations in $J_{i}(t)$ is high enough to hardly influence the time integral. The striking thing is, for all the other data where the effect of the oscillations is not negligible, the time integrals clearly 


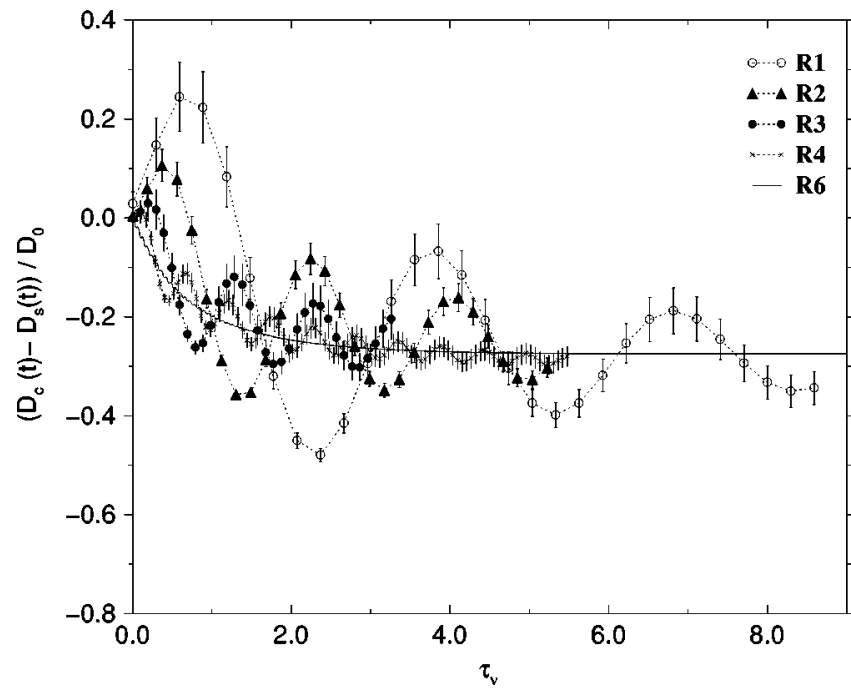

FIG. 8. The time integral of the interaction correlation function, made dimensionless by dividing the Stokes-Einstein diffusion coefficient $D_{0}$. The reduced wave vector $k^{*}$ was equal to 0.314 . Data for system $R 5$ are omitted for clarity.

oscillate about the same underlying function as run $R 6$. This is despite the fact that between runs $R 1$ and $R 6$ the compressibility factor varies by a factor of 50 . It is therefore clear that the correlation function consists of a compressible component, which oscillates about zero and ultimately contributes nothing to the integral, and an incompressible component, determined entirely by $\tau_{\nu}$. Although we only show results for $k^{*}=0.314$, where the statistical errors are minimal, the same was true for all the wave vectors we studied.

\section{DISCUSSION}

Having carefully examined the role sound plays in propagating hydrodynamic interactions, we arrive at the following conclusions. On the dynamics of a single tagged particle in a suspension, the effect of sound is relatively trivial. Sound propagation only influences the dynamics on time scales of the order of the time it takes a sound wave to propagate a distance the order of a particle radius.

If, however, we consider collective motions, that is the ability of one particle to influence the velocity of others, the situation is more complicated. We observe oscillations in the current-current correlation function that are clearly the result of sound propagation back and forth between particles. These oscillations are not restricted to the very short time scale characteristic of sound propagating a characteristic distance in the suspension. Rather, in the limit of small wave vectors $(k \ll 1 / a)$, these oscillations will be negligible, our results imply, only when $t \gg k^{2} \Gamma$. Thus, at sufficiently small wave vectors, the influence of sound propagation on the correlation function can always be significant. If we ask the question, do hydrodynamic interactions propagate at the speed of sound, the answer is a qualified yes. Strictly, a part of the time-dependent interaction of one particle with its neighbors is governed by the speed of sound and the sound wave attenuation coefficient. Since these are quantities characteristic of sound propagation, this clearly implies a sonic propagation of hydrodynamic interactions. Thus, our results show that the motion of one particle does influence others by sound propagation. However, we might prefer to define a hydrodynamic interaction as the motion of one particle influencing another on average. If we took this definition we would come to a different conclusion. Looking at the integrated effects of sound propagation we found no net effect for times greater than the oscillation period. On average, sound does nothing but induce wiggles about the incompressible result. It is still, therefore, the viscous diffusion mechanism that determines the time scale on which the interactions have a net effect-the "relaxation" time scale on which the transport coefficients are asymptotic. Consequently, we find no evidence to support the speed of sound propagation mechanism suggested by Ladd et al. to explain the different relaxation times observed in computer simulations and experiment. The origin of this discrepancy remains unknown.

Where our simulations show that sound propagation is relevant, its role can, we suggest, be understood relatively simply. At sufficiently small wave vectors (the simulations suggest roughly $k a<0.1$ ) the effect of sound in a suspension is the same as the effect of sound propagation in a simple fluid. In other words, if one views a colloidal suspension on length scales very much longer than the size of the particles, it behaves in the same way as a generic "structureless" fluid. This can be understood quite simply in terms of what one may term classical hydrodynamics. The only modification required is that the transport coefficients be those characteristic of the suspension (speed of sound, bulk- and shear viscosity), rather than the solvent. Our simulations suggested that for a suspension where the colloidal particles occupy $25 \%$ of the available volume, the speed of sound is approximately 1.05 times the value for the solvent and the bulk viscosity 4.8 times the solvent value. Relative to other transport coefficients, this implies a very weak dependence on volume fraction for the former and a very strong dependence for the latter. The scattering of sound waves by the particles is, it seems, a more effective means of dissipating density fluctuations than propagating them.

Based on these results we can estimate when soundinduced oscillations should be observable experimentally. To do so we return to the time-dependent transport coefficients. The quantity measured experimentally, in photocorrelation spectroscopy, for example, is the dynamic structure factor $S(k, t)$. The decay of the dynamic structure factor, to lowest order in $k$, is given by

$$
S(k, t)=S(k, 0) \exp \left(-k^{2} \int_{0}^{t} D\left(k, t^{\prime}\right) d t^{\prime}\right),
$$

where

$$
D(k, t)=\int_{0}^{t} J\left(k, t^{\prime}\right) d t^{\prime} .
$$

If we approximate the nonoscillatory components of $J(k, t)$ by their asymptotic values (valid for times $t>a^{2} / \nu$ ), we have 


$$
\int_{0}^{t} D(k, t) \simeq D(k, t) t+\int_{0}^{t} \int_{0}^{t^{\prime}} J_{i}^{s}\left(k, t^{\prime \prime}\right) d t^{\prime \prime} d t^{\prime} .
$$

In order for the oscillations in the second term to be experimentally observable, the condition

$$
\frac{\int_{0}^{t} \int_{0}^{t^{\prime}} J_{i}^{s}\left(k, t^{\prime \prime}\right) d t^{\prime \prime} d t^{\prime}}{D_{0} t} \sim 1,
$$

where $D_{0}$ is the Stokes-Einstein diffusion coefficient, should be satisfied. Substituting the hydrodynamic result for $J_{i}^{s}(k, t)$ [Eq. (7)] and performing the double integral, this corresponds to the condition

$$
t \sim \frac{\nu}{\left(c k^{*}\right)^{2}}
$$

We have also taken the limit $k^{*} \ll \alpha$, but, as we have seen, for any colloidal suspension the compressibility factor $\alpha$ is greater than unity so this only corresponds to the condition $k^{*}<1$ (required anyway for the simple hydrodynamic result for the oscillatory component of the correlation function to be valid). The conclusion we therefore arrive at is that sound-induced oscillations in the dynamic structure factor should be observable at short times. To see how short, we can substitute values for $c$ and $\nu$ typical of water at room temperature. This yields $t<10^{-10} s$ at $k^{*}=10^{-1}$, which is rather short, or more reasonably $t<10^{-6} s$ at $k^{*}=10^{-3}$. We should also point out that the dynamic structure factor decays on a time scale $t \sim 1 /\left(k^{2} D(k)\right)$, and that unless the colloidal particles are very small $(a \sim 1 \mathrm{~nm})$, the time regime defined by Eq. (17) is very much shorter. Thus, any effect of sound propagation on the dynamic structure factor would manifest itself more as an oscillation about the initial value than a modulation of the subsequent decay.

\section{ACKNOWLEDGMENTS}

The research of C.P.L. has been made possible by a fellowship of the Royal Netherlands Academy of Arts and Sciences. The authors are grateful to Andrew Masters and Paul Bartlett for their useful comments.
${ }^{1}$ S. T. Milner and A. J. Liu, Phys. Rev. E 48, 449 (1993).

${ }^{2}$ H. J. H. Clercx, Phys. Rev. E 56, 2950 (1997).

${ }^{3}$ M. H. J. Hagen, I. Pagonabarraga, D. Frenkel, and C. P. Lowe, Phys. Rev. Lett. 78, 3785 (1997).

${ }^{4}$ I. Pagonabarraga, M. H. J. Hagen, C. P. Lowe, and D. Frenkel, Phys. Rev. E 59, 4458 (1999).

${ }^{5}$ A. J. C. Ladd, H. Gang, J. X. Zhu, and D. A. Weitz, Phys. Rev. E 52, 6550 (1995).

${ }^{6}$ A. J. C. Ladd, H. Gang, J. X. Zhu, and D. A. Weitz, Phys. Rev. Lett. 74, 318 (1995).

${ }^{7}$ S. Henderson, S. Mitchell and P. Bartlett, Colloids Surf., A (in press).

${ }^{8}$ H. Mori, Prog. Theor. Phys. 34, 399 (1965).

${ }^{9}$ B. J. Berne and G. D. Harp, Adv. Chem. Phys. 17, 63 (1970).

${ }^{10}$ J. M. Deutch and I. Oppenheim, Faraday Discuss. Chem. Soc. 83, 1 (1987)

${ }^{11} \mathrm{~J}$. A. McLennan, Introduction to Non-equilibrium Statistical Mechanics (Prentice-Hall, Englewood Cliffs, NJ, 1989).

${ }^{12}$ D. Bedeaux and P. Mazur, Physica (Amsterdam) 78, 505 (1974).

${ }^{13}$ A. J. Masters, Mol. Phys. 57, 303 (1986).

${ }^{14}$ J. X. Zhu, D. J. Durian, J. Müller, D. A. Weitz, and D. J. Pine, Phys. Rev. Lett. 68, 2559 (1992).

${ }^{15}$ M. H. Kao, A. G. Yodh, and D. J. Pine, Phys. Rev. Lett. 70, 242 (1993).

${ }^{16}$ A. J. C. Ladd, Phys. Rev. Lett. 70, 1339 (1993).

${ }^{17}$ A. J. C. Ladd, J. Fluid Mech. 271, 311 (1994).

${ }^{18}$ C. P. Lowe and D. Frenkel, Phys. Rev. E 54, 2704 (1996).

${ }^{19}$ P. Español, M. A. Rubio, and I. Zúñiga, Phys. Rev. E 51, 803 (1995).

${ }^{20}$ P. Español, Physica A 214, 185 (1995).

${ }^{21}$ A. J. C. Ladd, J. Fluid Mech. 271, 285 (1994).

${ }^{22}$ G. R. McNamara and B. J. Alder, in Microscopic Simulation of Complex Hydrodynamic Phenomena, edited by M. Mareschal and B. L. Holian (Plenum, New York, 1992).

${ }^{23}$ C. P. Lowe, D. Frenkel, and A. J. Masters, J. Chem. Phys. 103, 1582 (1995).

${ }^{24}$ M. W. Heemels, M. H. J. Hagen, and C. P. Lowe, J. Comput. Phys. 164, 48 (2000).

${ }^{25}$ M. Hagen, D. Frenkel, and C. P. Lowe, Physica A 272, 376 (1999).

${ }^{26}$ C. W. J. Beenakker and P. Mazur, Physica A 128A, 48 (1984).

${ }^{27}$ A. J. C. Ladd, J. Chem. Phys. 93, 3484 (1990).

${ }^{28}$ J.-P. Hansen and I. R. McDonald, The Theory of Simple Liquids (Academic, London, 1986) (McGraw-Hill, New York, 1980).

${ }^{29}$ J.-P. Boon and S. Yip, Molecular Hydrodynamics (McGraw-Hill, New York 1980).

${ }^{30}$ K. Sköld and K. E. Larsson, Phys. Rev. 161, 102 (1967).

${ }^{31}$ A. Rahman, in Neutron Inelastic Scattering 1 (International Atomic Energy Agency, Vienna, 1968).

${ }^{32}$ C.-H Chung and S. Yip, Phys. Lett. 50A, 175 (1974).

${ }^{33}$ A. J. C. Ladd, J. Chem. Phys. 93, 3484 (1990). 